\title{
- Platelet studies in patients with coronary artery disease and in their relatives
}

\author{
J. R. Hampton and R. Gorlin \\ - From the Department of Medicine, Nottingham University; and the Cardiovascular Division, \\ Peter Bent Brigham Hospital, Boston, Massachusetts, U.S.A.
}

Measurement of the platelet electrophoretic response to adenosine diphosphate $(A D P)$ has been used to study platelets from two groups of patients thought to have coronary artery disease, and - a group of relatives of men who had suffered myocardial infarctions before the age of 45 .

Of 34 patients diagnosed by clinical, biochemical, and electrocardiographic criteria to have had myocardial infarctions, 32 had platelets that were abnormally sensitive to ADP. The coronary arteries of 12 further patients with chest pain were assessed by angiography: of these, 8 were found to have coronary artery disease and 4 had normal coronary arteries. Seven of the 8 patients with - abnormal coronary arteries had platelets that were abnormally sensitive to ADP; one of the 4 patients with normal coronary arteries had abnormal platelets.

Of 29 relatives of young men with arterial disease, 17 had platelets that were abnormally sensitive to ADP. Of 107 subjects studied in the course of other experiments, 3 were found to have platelets that showed this abnormality.

- Studies of platelet function have not so far been of clinical value in the diagnosis of coronary

2. artery disease, nor have they been found useful in the identification of individual subjects destined to develop it. Platelet adhesiveness (the sticking of platelets to a foreign surface such as glass) is increased in patients with coronary artery disease (McDonald and Edgill, 1957) but changes in platelet adhesiveness are - non-specific, and they have been observed in a wide variety of other conditions. An increase

1 - in platelet aggregation (the sticking of platelets to each other) has also been described in patients with coronary artery disease (O'Brien, Heywood, and Heady, 1966) but such a

- change is also non-specific, for it occurs after surgical operations (Emmons and Mitchell, 1965); moreover, differences in platelet aggre-

- gation between healthy and ill subjects can only be detected when groups are compared because of the wide variation in platelet aggregation.

Measurement of the changes in platelet electrophoretic mobility induced by adenosine

4 diphosphate (ADP) and noradrenaline has been found more useful clinically, for the platelets of patients who have had myocardial infarction are unusually sensitive to ADP. ADP and noradrenaline induce biphasic chan-

Received 7 July 1971. ges in platelet mobility: the maximum mobility that can be induced is about ro per cent above the control value and in healthy subjects this increase is induced by $0.05 \mu \mathrm{g} / \mathrm{ml}$ of either ADP or noradrenaline (Hampton and Mitchell, 1966a). In patients with any sort of acute illness the maximum increase in platelet mobility is induced by $0.005 \mu \mathrm{g} / \mathrm{ml}$ (or less) of ADP or noradrenaline (Hampton and Mitchell, 1966b). In patients with atheromatous arterial disease platelet sensitivity to ADP is increased so that maximum platelet mobility is induced by $0.005 \mu \mathrm{g} / \mathrm{ml}$, while the platelet sensitivity to noradrenaline is normal (Hampton and Mitchell, 1966c). In these patients the change in platelet behaviour results from a disturbance of the plasma lipids, the nature of which has only partly been elucidated (Bolton, Hampton, and Mitchell, 1967). A similar abnormality in the plasma lipids and the platelets has been described in women taking oral contraceptives (Bolton, Hampton, and Mitchell, 1968), but it appears to be limited to these two situations. Pending the final clarification of the plasma lipid abnormality, observations of the platelet electrophoretic behaviour of patients with arterial disease have been extended, and a group of subjects whose coronary arteries were investigated by angiography has been studied. Observations have also been made of the 
platelet behaviour of a group of close relatives of patients who developed myocardial infarctions before the age of 45 .

\section{Methods}

Blood was collected by clean venepuncture into plastic syringes, and $9 \mathrm{ml}$ aliquots were transferred to siliconized centrifuge tubes containing I ml of 3.8 per cent $(\mathrm{w} / \mathrm{v})$ trisodium citrate. Platelet-rich plasma and platelet-poor plasma were prepared and the platelet-rich plasma was diluted $I$ in Io with the platelet-poor plasma. Measurements of platelet mobility were made in a horizontal capillary apparatus with platinum electrodes using the technique described by Hampton and Mitchell (1966a). Adenosine diphosphate and noradrenaline (both from Sigma) were dissolved in saline, and platelet mobilities were measured before and after ro minutes incubation with $0.005,0.05$, and $0.5 \mu \mathrm{g} / \mathrm{ml}$.

\section{Results}

(a) Preliminary study: patients with clinically-diagnosed coronary artery disease Thirty-four patients, 28 men (aged 27-70) and 6 women (aged 34-77) were studied. All but 2 had suffered myocardial infarctions, diagnosed by clinical history and signs, changes in the electrocardiogram, changes in plasma enzymes, and changes in the peripheral white cell count and the red cell sedimentation rate. Patients in whom the diagnosis was in doubt were not studied. No patient was studied within two weeks of a myocardial infarction and no patient was studied if he was suffering from any illness that might lead to a nonspecific increase in platelet sensitivity to both ADP and noradrenaline. The two patients who were included in this group but who had not suffered myocardial infarctions had had severe exertional chest pain which was characteristic of angina pectoris; in both the electrocardiogram showed horizontal depression of the ST segments in the anterior chest leads.

In the first 20 patients the platelets were exposed to ADP and to noradrenaline and matched control subjects were also studied: the detailed results have been described previously (Hampton and Mitchell, 1966c). The platelets of the remaining 14 patients were exposed only to ADP. Fig. I shows the effects of ADP on the platelets of all 34 patients: the interrupted line and stippled area show the mean changes in mobility and their standard deviations in 31 patients in whom the maximum platelet mobility was induced by 0.005 $\mu \mathrm{g} / \mathrm{ml}$ of ADP, and the response curves of the platelets from the other 3 patients are shown by individual lines. In 2 of these patients the platelets appeared normally sensitive to ADP,

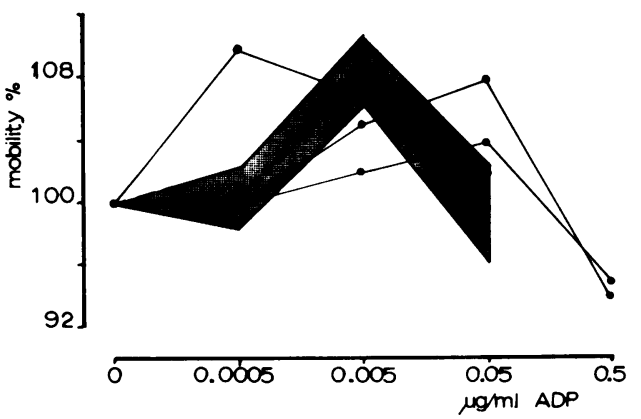

FIG. I Platelet mobility changes induced by adenosine diphosphate in patients with clinically diagnosed coronary artery disease. Interrupted line represents mean mobilities of 31 subjects and stippled area shows standard deviation; continuous lines represent 3 individual subjects.

maximum mobility being induced by 0.05 $\mu \mathrm{g} / \mathrm{ml}$. One patient had platelets which were extremely sensitive to ADP, maximum platelet mobility being induced by $0.0005 \mu \mathrm{g} / \mathrm{ml}$.

(b) Patients with clinically-diagnosed coronary artery disease who were assessed by coronary angiography The results from the preliminary study suggest that platelet electrophoretic behaviour provides a method for discriminating patients with myocardial infarctions from healthy subjects. However, it has recently been suggested that in many patients coronary artery disease cannot confidently be diagnosed unless coronary angiography is performed. From a large group of patients with sufficiently severe chest pain to require investigation by coronary angiography, Kemp, Elliott, and Gorlin (1967) described 50 patients whose coronary angiograms were normal. A similar report was published by Likoff, Segal, and Kasparian (1967), and patients have also been described who had suffered myocardial infarctions well documented by the usual criteria, but whose coronary arteries were subsequently found to be entirely normal (Sidd, Kemp, and Gorlin, 1970). It is possible that the two patients described above who apparently had had myocardial infarctions but whose platelets were normal did not have diseased coronary arteries.

Platelet electrophoretic behaviour was therefore studied in 12 patients with severe chest pain in whom coronary angiography was to be performed. The group included 8 men (aged 34-48) and 4 women (aged 42-56): 6 of these patients were thought to have had previous myocardial infarctions, 5 had severe exertional chest pain, and one had chest pain 
$\therefore$ but investigation was performed primarily because a bad family history of heart disease had induced a severe anxiety state. In each case the study of platelet electrophoretic behaviour was performed before angiography so as to avoid any changes in platelet behaviour that might result from the angiogra-

- phic procedure, and so that measurements of platelet mobility would not be biased by

' knowledge of the state of the patient's coronary arteries. The coronary angiograms were performed by the technique of Sones and Shirey (1962), and they were reviewed by an experienced observer (Dr. H. G. Kemp) who

was unaware of the results of the platelet studies. Disease of the right and left main coronary arteries (and of the left anterior descending and left circumflex branches) was classified as complete occlusion, or severe ( $75 \%$ or more), moderate $(25-75 \%)$, or mild (less than $25 \%$ ) stenosis.

- Patients with abnormal coronary angiograms

- Eight of the patients studied were found to have diseased coronary arteries. Of these, 7 had platelets which were abnormally sensitive to ADP but normally sensitive to noradrenaline. In 6 of these patients the maximum platelet mobility was induced by $0.005 \mu \mathrm{g} / \mathrm{ml}$ 1 of ADP, and in the other maximum mobility was induced by $0.0005 \mu \mathrm{g} / \mathrm{ml}$. The platelets of the eighth patient showed normal sensitivity to ADP, maximum mobility being induced by $0.05 \mu \mathrm{g} / \mathrm{ml}$. In Fig. 2 the stippled area includes the mobility responses to ADP of the 6 patients in whom maximum platelet mobility was induced by $0.005 \mu \mathrm{g} / \mathrm{ml}$; the

$\sim$

- FIG. 2 Platelet mobility changes induced by adenosine diphosphate in 8 patients with abnormal coronary arteriograms.

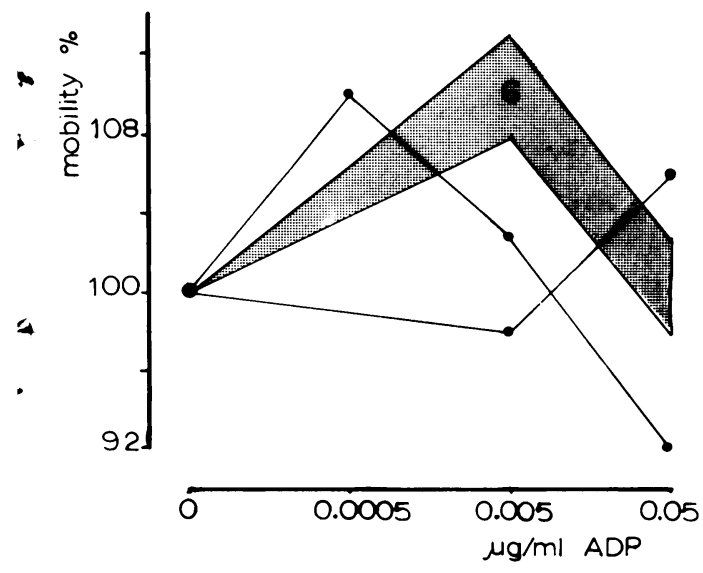

other two response curves are shown separately.

The 6 patients in whom maximum platelet mobility was induced by $0.005 \mu \mathrm{g} / \mathrm{ml}$ of ADP were all men; 5 of them had previously been admitted to hospital with myocardial infarctions. Two of these 5 patients had disease of the right coronary artery, the left anterior descending branch, and the left circumflex branch; one had complete occlusion of the left anterior descending branch and severe stenosis of the right coronary artery; one had severe stenosis of the right coronary artery; and one had moderate stenosis only of the right coronary artery. Two of these 5 patients had abnormal blood lipids, with the pattern characterized by Fredrickson and Lees (1965) as Type IV. The sixth patient in this group had severe exertional chest pain but he was not thought to have had a myocardial infarction: angiography revealed complete occlusion of both the right coronary artery and the left circumflex branch, and severe stenosis of the left anterior descending branch.

The patient in whom maximum platelet mobility was induced by $0.0005 \mu \mathrm{g} / \mathrm{ml}$ of ADP was also thought to have angina, but he did not appear to have had a myocardial infarction. Angiography showed regions of moderate stenosis in the left anterior descending branch, and two such regions in the right coronary artery.

The only patient whose platelets were normal but whose coronary angiograms were abnormal was a woman aged 49: her left coronary system was entirely normal but her right coronary artery showed mild irregularities.

\section{Patients with normal coronary angiograms}

Fig. 3 shows the platelet response to ADP of 4 patients whose coronary angiograms were normal. In 3 of these patients the platelet response was normal, maximum mobility being induced by $0.05 \mu \mathrm{g} / \mathrm{ml}$; the stippled area in Fig. 3 includes the mobility curves of these patients. One patient with normal coronary angiograms had platelets that were abnormally sensitive to ADP and the response of these platelets is shown separately.

Table I shows the numbers of patients with normal and abnormal platelet behaviour in the group of patients diagnosed to have coronary artery disease by clinical and electrocardiographic criteria, and in the group studied with coronary angiography.

(c) Relatives of young patients with arterial disease The abnormalities in platelet behaviour described could precede or 

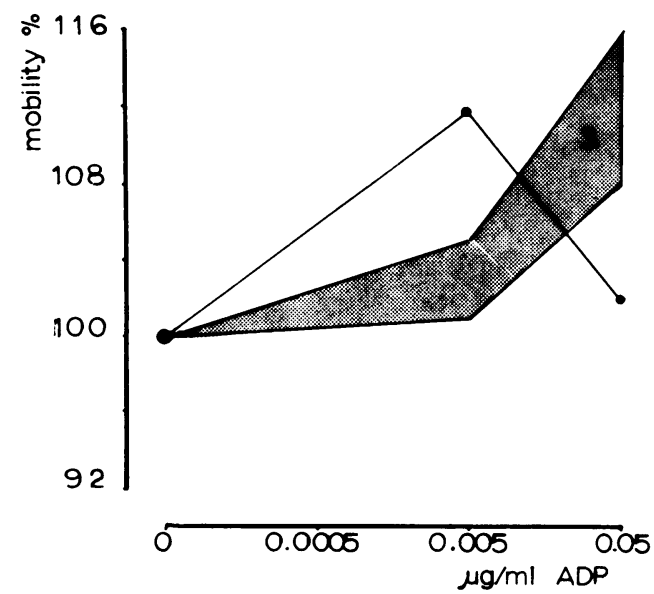

FIG. 3 Platelet mobility changes induced by adenosine diphosphate in 4 patients with normal coronary arteriograms.

result from arterial disease, or they could merely occur in parallel with it. One method of determining whether platelet abnormalities occur before the development of arterial disease is to study groups of subjects who are known to be more liable to develop it than the general population. Patients with hyperlipidaemia are known to have a high risk of myocardial infarction, and such patients do have platelets that are abnormally sensitive to ADP (Hampton and Mitchell, 1966c). Another group of subjects at risk are the relatives of young patients with myocardial infarctions (Deutscher, Ostrander, and Epstein, 1970).

Platelet electrophoretic behaviour was therefore investigated in 29 close relatives of I4 patients who had developed clinical manifestations of arterial disease before the age of 45. Of these 14 patients, 13 had had welldocumented myocardial infarctions, and one had severe peripheral vascular disease. No attempt was made to study the platelets of the patients themselves, as some had died and others were acutely ill.

TABLE I Platelet electrophoretic response to $A D P$ in patients with chest pain

\begin{tabular}{lll}
\hline & \multicolumn{2}{c}{$\begin{array}{l}\text { Platelet sensitivity } \\
\text { to ADP }\end{array}$} \\
\cline { 2 - 3 } & Normal & Abnormal \\
\hline $\begin{array}{c}\text { Patients with clinically diag- } \\
\text { nosed coronary artery disease } \\
\begin{array}{c}\text { Patients with abnormal } \\
\text { coronary angiograms }\end{array}\end{array}$ & 2 & 32 \\
$\begin{array}{c}\text { Patients with normal coronary } \\
\text { angiograms }\end{array}$ & I & 7 \\
\hline
\end{tabular}

The 29 relatives comprised one father, 17 children, 6 sibs, and 5 children of these sibs. The presence of vascular disease in these subjects was excluded as far as possible by history, clinical examination, and electrocardiography. Whenever possible, fasting serum samples were taken for cholesterol, triglyceride, and lipoprotein electrophoresis measurements.

The effects of 0.005 and $0.05 \mu \mathrm{g} / \mathrm{ml}$ of ADP on platelet mobility were studied in each case. Whenever possible the effects of noradrenaline were also determined, but the difficulty of obtaining sufficient blood prevented this in several of the children. In all cases care was taken to avoid studying any subject who was ill in any way, so as to avoid the changes in platelet behaviour caused by acute illness.

The response of the platelets of each subject was classified as 'normal' if $0.05 \mu \mathrm{g} / \mathrm{ml}$ of ADP induced a greater increase in mobility than did $0.005 \mu \mathrm{g} / \mathrm{ml}$ and 'abnormal' if 0.005 $\mu \mathrm{g} / \mathrm{ml}$ induced a greater increase than 0.05 $\mu \mathrm{g} / \mathrm{ml}$. These definitions are of necessity artificial, for the maximum mobility might be induced by an intermediate concentration of ADP. Fig. 4 shows the response to ADP and noradrenaline of the 22-year-old daughter of a man who died of a myocardial infarction at the age of $45: 0.05 \mu \mathrm{g} / \mathrm{ml}$ of noradrenaline increased platelet mobility to 16 per cent above the initial level, which approximates to the maximum mobility that would be expected (Hampton and Mitchell, 1966d). Incubation with 0.005 and $0.05 \mu \mathrm{g} / \mathrm{ml}$ of ADP induced a 2 per cent and a 4 per cent increase, respectively, so by definition the response of the platelets to ADP was normal: but it seems likely that the maximum mobility would have been induced by a concentration of ADP somewhere between 0.005 and $0.05 \mu \mathrm{g} / \mathrm{ml}$, as indicated by the dotted line in Fig. 4. However, because of the technical problems of testing intermediate concentrations we decided to accept the restrictions imposed by these definitions of 'normality' and 'abnormality'.

Of the 29 subjects studied, 17 had platelets that were abnormally sensitive to ADP. Of these 17, II were male and 6 were female; their mean age was 25 years (SD I4 years). of the 12 subjects with normal platelet sensitivity to ADP, 7 were male and 5 were female; the mean age of this group was 16 years (SD 8 years). Of the children studied who were aged 14 or less, 6 had normal platelets and one (aged II) had abnormal platelets.

Of the 17 subjects with abnormal platelet behaviour, the relationships to the patients with arterial disease were as follows: one was the 53-year-old father of a man who had a 


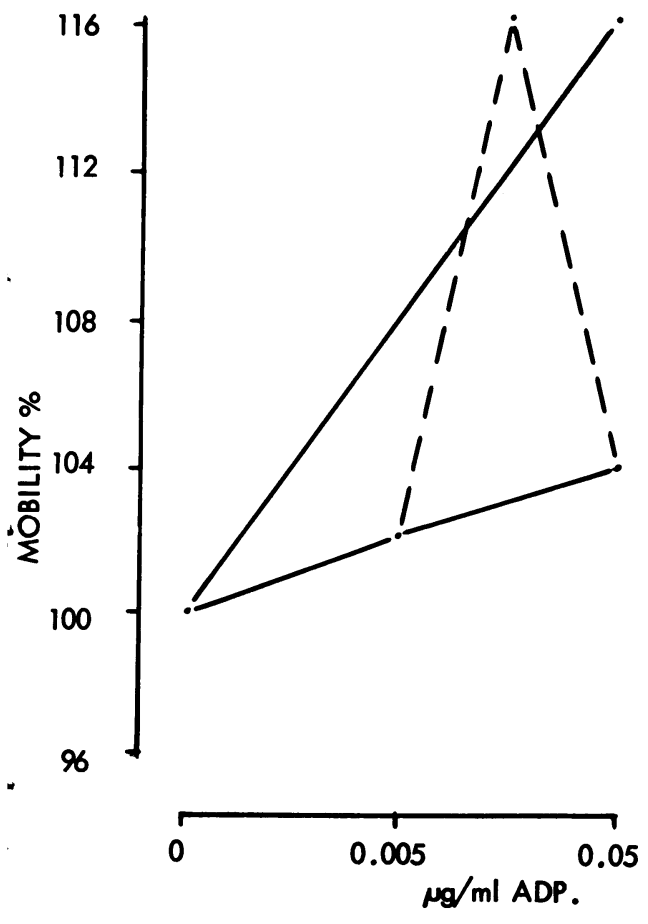

FIG. 4 Platelet mobility changes induced by adenosine diphosphate and noradrenaline in a \woman aged 22 whose father died of a myocardial infarction (see text).

myocardial infarction at the age of $27 ; 5$ were sibs (4 male and I female); 9 were children; and 2 were children of sibs whose platelets were abnormal. Of the 12 subjects whose platelets were normal, one was a sib (male), , 8 were children, and 3 were children of sibs whose platelets were abnormal.

- Table 2 shows that there were no significant differences between the subjects with normal and abnormal platelets in terms of serum levels of cholesterol or triglycerides; there were also no differences in the frequency of abnormal lipoprotein electrophoresis. Two of the children studied were of particular interest

TABLE 2 Platelet electrophoretic response to $A D P$ and plasma lipids in relatives of young men with arterial disease

\begin{tabular}{lll}
\hline & \multicolumn{2}{c}{ Platelet sensitivity to ADP } \\
\cline { 2 - 3 } & Normal & Abnormal \\
\hline $\begin{array}{c}\text { Mean cholesterol } \\
(\mathrm{mg} / \mathrm{r} 00 \mathrm{ml} \pm \text { SEM) }\end{array}$ & $194 \pm 20$ & $219 \pm$ ro \\
$\begin{array}{c}\text { Mean triglycerides } \\
(\mathrm{mg} / \mathrm{100} \mathrm{ml} \pm \text { SEM) }\end{array}$ & $156 \pm 30$ & $157 \pm 24$ \\
\hline
\end{tabular}

with respect to their blood lipids. These were the 19 and 17 -year-old sons of a patient who had sustained three myocardial infarctions by the age of 45 . The family had been studied five years previously, and several members with hyperlipidaemia had been identified. At that time the serum cholesterol levels of these boys (then aged 14 and 12 ) were 295 and 310 $\mathrm{mg} /$ I00 $\mathrm{ml}$, respectively. They had been treated by dietary modification and the diet had been adhered to rigorously; at the time their platelets were studied their serum cholesterol levels were both $195 \mathrm{mg} / 100 \mathrm{ml}$, and yet both of them showed abnormal platelet behaviour.

(d) Platelet electrophoretic behaviour in an unselected population No attempt has been made to study as a control group the healthy relatives of subjects without arterial disease, largely because of the difficulty of obtaining sufficiently large blood samples from children. However, during the course of other experiments, the effects of ADP 0.005 and $0.05 \mu \mathrm{g} / \mathrm{ml}$ on platelet mobility have been studied in 107 subjects who were expected to have normal platelets. Of these, 45 were healthy volunteers (mainly medical and laboratory staff, medical students, and nurses) and 62 were hospital inpatients who were not acutely ill but who had a very wide variety of nonvascular conditions. A total of I04 of these subjects had platelets which behaved normally, $0.05 \mu \mathrm{g} / \mathrm{ml}$ of ADP inducing a larger increase in mobility than $0.005 \mu \mathrm{g} / \mathrm{ml}$. Three subjects had platelets which were abnormally sensitive to ADP, $0.005 \mu \mathrm{g} / \mathrm{ml}$ inducing a larger increase in mobility than 0.05 $\mu \mathrm{g} / \mathrm{ml}$. Each of these subjects was studied on at least three occasions, and the platelets were found to be abnormally sensitive to ADP each time. On at least one occasion the platelet sensitivity to noradrenaline was investigated and shown to be normal, thus excluding the possibility of the nonspecific increase in platelet sensitivity characteristic of any acute illness. Two of these subjects (a 28-year-old physician and a 22-year-old medical student) were apparently completely healthy; the third (a 48-year-old ambulance driver) was admitted to hospital with a peptic ulcer. None of these 3 had a family history of premature vascular disease.

\section{Discussion}

Most patients with coronary artery disease appear to have platelets that are abnormally sensitive to adenosine diphosphate in terms of their electrophoretic behaviour; many of 
the relatives of young patients with arterial disease show the same abnormality, and it is tempting to speculate that the platelet abnormality may precede and possibly even lead to the development of arterial disease. If this is true, then investigation of an individual subject's platelet electrophoretic behaviour can only be of limited value as a diagnostic aid for the investigation of vascular disease, for the finding of abnormal platelet behaviour would not indicate how far arterial disease had advanced. However, we intend to study the platelets of more patients undergoing coronary arteriography to find out whether normal platelet behaviour can be used as an indication of the lack of arterial disease; ultimately it may be possible to reduce the need for angiography in the relatively large number of patients who have chest pain that requires investigation but who are found to have normal coronary arteries.

It seems unlikely that all the relatives of patients with myocardial infarctions whose platelets were found to be abnormally sensitive to ADP will develop arterial disease early in life, and the value of platelet electrophoretic behaviour in the identification of individual subjects particularly at risk can only be determined by following for a prolonged period subjects whose platelets have been studied. If the platelet abnormality does precede the development of arterial disease then it might be expected that individuals with abnormal platelets would sometimes be encountered unexpectedly: in fact this occurred 3 times in I07 subjects whose platelets were expected to be normal and who were being studied for other purposes. A much larger group must be studied before the frequency of 'abnormal' platelet behaviour can be established, but it does seem that 'abnormal' platelets are found more often in the close relatives of young men with myocardial infarctions than in the general population (Table 3).

Observations of platelet electrophoretic behaviour appear to provide better discrimination between patients with arterial disease and healthy subjects than do measurements

TABLE 3 Platelet electrophoretic response to $A D P$ in relatives of young men with arterial disease and in unselected population

\begin{tabular}{lcc}
\hline & \multicolumn{2}{c}{ Platelet sensitivity to ADP } \\
\cline { 2 - 3 } & Normal & Abnormal \\
\hline Relatives & 12 & 17 \\
Unselected group & 104 & 3 \\
\hline
\end{tabular}

of platelet adhesiveness or platelet aggregation, but the main limitation to the clinical application of platelet electrophoresis lies in the need to separate 'normal' and 'abnormal' platelet behaviour according to the mobility change induced by 0.005 and $0.05 \mu \mathrm{g} / \mathrm{ml}$ of ADP. Though it is possible that there are two populations, one in which maximum platelet mobility is induced by ADP $0.05 \mu \mathrm{g} / \mathrm{ml}$ ("normal') and another in which maximum mobility is induced by ADP $0.005 \mu \mathrm{g} / \mathrm{ml}$ ('abnormal'), it seems much more likely that there is a continuous variation in the concentration of ADP required to induce maximum platelet mobility within the total population. It is not practicable to construct for each individual detailed mobility response curves with many concentrations of ADP and noradrenaline, because the technique is tedious and time-consuming, and relatively large volumes of blood are required. Clarification of the lipid abnormality responsible for the increased platelet sensitivity to ADP may lead to a simpler and more quantitative technique for the study of larger groups of subjects, and experiments to this end are in progress.

J. R. Hampton gratefully acknowledges support from a Wellcome Foundation travel grant, and the Edith Walsh Research Award from the British Medical Association. This study was partly supported by grants from the U.S.P.H.S. Undergraduate Training Grant programme, and four other U.S.P.H.S. grants. We are grateful to Dr. H. G. Kemp for reviewing the coronary angiograms, and to the physicians of the Radcliffe Infirmary, Oxford, the Nottingham General Hospital, and the Peter Bent Brigham Hospital, Boston, Massachusetts, for permission to study patients in their care.

\section{References}

Bolton, C. H., Hampton, J. R., and Mitchell, J. R. A. (1967). The nature of the transferable factor which causes abnormal platelet behaviour in vascular disease. Lancet, 2, I IOI.

Bolton, C. H., Hampton, J. R., and Mitchell, J. R. A. (I968). Effect of oral contraceptive agents on plate lets and plasma phospholipids. Lancet, I, 1336.

Deutscher, S., Ostrander, L. D., and Epstein, F. H. (1970). Familial factors in premature coronary heart disease - a preliminary report from the Tecumseh community health study. American fournal of Epidemiology, 91, 233.

Emmons, P. R., and Mitchell, J. R. A. (1965). Postoperative changes in platelet-clumping activity. Lancet, $\mathbf{r}, 7 \mathbf{1}$.

Fredrickson, D. S., and Lees, R. S. (1965). A system for phenotyping hyperlipoproteinaemia. Circulation, 31, 32I.

Hampton, J. R., and Mitchell, J. R. A. (1966a). Effect of aggregating agents on the electrophoretic mobility of human platelets. British Medical fournal, r, 1074 
Hampton, J. R., and Mitchell, J. R. A. (1966b). Abnormalities in platelet behaviour in acute illnesses. British Medical fournal, $\mathrm{x}, \mathrm{1078.}$

Hampton, J. R., and Mitchell, J. R. A. (I966c). A transferable factor causing abnormal platelet behaviour in vascular disease. Lancet, 2, 764.

Hampton, J. R., and Mitchell, J. R. A. (1966d). An estimate of the number of adenosine diphosphate binding sites on human platelets. Nature (London), 21 I, 245.

' Kemp, H. G., Elliott, W. C., and Gorlin, R. (1967). The anginal syndrome with normal coronary arteriography. Transactions of the Association of American Physicians, 80, 59.

Likoff, W., Segal, B. L., and Kasparian, H. (1967). Paradox of normal selective coronary arteriograms in patients considered to have unmistakable coronary heart disease. New England fournal of Medicine, 276, 1063.

McDonald, L., and Edgill, M. (1957). Coagulability of the blood in ischaemic heart-disease. Lancet, 2, 457.

O'Brien, J. R., Heywood, J. B., and Heady, J. A. (1966). The quantitation of platelet aggregation induced by four compounds: a study in relation to myocardial infarction. Thrombosis et diathesis haemorrhagica, 16, 752.

Sidd, J. J., Kemp, H. G., and Gorlin, R. (1970). Acute myocardial infarction in a nineteen-year-old student in the absence of coronary obstructive disease. New England Fournal of Medicine, 282, 1306.

Sones, F. M., and Shirey, E. K. (1962). Cine coronary angiography. Modern Concepts of Cardiovascular Disease, 31, 735.

Requests for reprints to Dr. J. R. Hampton, Department of Medicine, General Hospital, Nottingham, NGI 6HA. 